\title{
Retinotopic Organization and Functional Subdivisions of the Human Lateral Geniculate Nucleus: A High-Resolution Functional Magnetic Resonance Imaging Study
}

\author{
Keith A. Schneider, ${ }^{1,2}$ Marlene C. Richter, ${ }^{2}$ and Sabine Kastner ${ }^{1,2}$ \\ ${ }^{1}$ Department of Psychology and ${ }^{2}$ Center for the Study of Brain, Mind and Behavior, Princeton University, Princeton, New Jersey 08544
}

\begin{abstract}
Functional magnetic resonance imaging (fMRI) has provided intriguing insights into the topography and functional organization of visual cortical areas in the human brain. However, little is known about the functional anatomy of subcortical nuclei. Here, we used high-resolution fMRI $\left(1.5 \times 1.5 \times 2 \mathrm{~mm}^{3}\right)$ at 3 tesla to investigate the retinotopic organization of the human lateral geniculate nucleus (LGN). The central $15^{\circ}$ of the visual field were mapped using periodic flickering checkerboard stimuli that evoked a traveling wave of activity. The contralateral visual hemifield was represented with the lower field in the medial-superior portion and the upper field in the lateral-inferior portion of each LGN. The horizontal meridian was significantly overrepresented relative to the vertical meridian. The fovea was represented in posterior and superior portions, with increasing eccentricities represented more anteriorly. The magnification of the fovea relative to the periphery was similar to that described for human primary visual cortex. The magnocellular regions of the LGN were distinguished based on their sensitivity to low stimulus contrast and tended to be located in its inferior and medial portions. Our results demonstrate striking similarities in the topographic organization of the macaque and human LGN and support accounts of a constant magnification from the retina through the cortex in both species.
\end{abstract}

Key words: lateral geniculate nucleus; retinotopy; magnification factor; magnocellular; parvocellular; luminance contrast

\section{Introduction}

The lateral geniculate nucleus (LGN) is the thalamic station in the projection of the visual pathway from retina to primary visual cortex (V1). Its topographic organization and the response properties of its neurons have been extensively studied in nonhuman primates (Polyak, 1953; Kaas et al., 1972; Malpeli and Baker, 1975; Connolly and Van Essen, 1984). The LGN is typically organized into six main layers, each of which receives input from either the contralateral or ipsilateral eye and contains a retinotopic map of the contralateral hemifield. The four dorsal layers contain small [parvocellular $(\mathrm{P})$ ] neurons characterized by sustained discharge patterns and low contrast gain, and the two ventral layers contain large [magnocellular $(\mathrm{M})$ ] neurons characterized by transient discharge patterns and high contrast gain (Wiesel and Hubel, 1966; Dreher et al., 1976; Creutzfeldt et al., 1979; Shapley et al., 1981; Derrington and Lennie, 1984; Merigan and Maunsell, 1993).

The contralateral visual hemifield is represented in the LGN with the horizontal meridian dividing the structure into a superior and medial half, representing the lower visual field, and an

\footnotetext{
Received June 18, 2004; revised Aug. 20, 2004; accepted Aug. 20, 2004.

This work was supported by Grants R01MH-64043 and P50MH-62196 from the National Institute of Mental Health and the Whitehall Foundation. We thank H. Fehd for assistance and D. Beck and T. Emmanouil for comments on this manuscript.

Correspondence should be addressed to Keith Schneider, Department of Psychology, Green Hall, Princeton University, Princeton, NJ 08544.E-mail: kas@princeton.edu.

DOI:10.1523/JNEUROSCI.2413-04.2004

Copyright $\odot 2004$ Society for Neuroscience $\quad 0270-6474 / 04 / 248975-11 \$ 15.00 / 0$
}

inferior and lateral half, representing the upper visual field. The fovea is represented medially in the posterior pole of the nucleus, whereas more peripheral visual field representations are located more anteriorly and laterally (Malpeli and Baker, 1975). In the LGN, as in other visual areas, more neurons are devoted to the representation of the fovea than to an equivalent area of the visual periphery. This distortion can be parameterized by an eccentricity magnification factor (Talbot and Marshall, 1941; Daniel and Whitteridge, 1961). In the macaque, it is not clear whether the relative representation of the fovea expands progressively from the retina through the LGN and V1 (Malpeli and Baker, 1975; Myerson et al., 1977; Connolly and Van Essen, 1984; Van Essen et al., 1984; Perry and Cowey, 1985; Azzopardi and Cowey, 1996) or is preserved throughout the visual hierarchy with no additional magnification present at the level of the LGN or V1 (Webb and Kaas, 1976; Schein and de Monasterio, 1987; Wässle et al., 1989, 1990; Malpeli et al., 1996).

In the human LGN, anatomical studies have revealed a similar organization compared with the macaque LGN in terms of laminar patterns. The layout of the representation of the visual field, however, is less well understood, because its study has historically been restricted to postmortem anatomical analyses of degeneration patterns after retinal or cortical lesions (Rönne, 1910; Juba and Szatmári, 1937; Kupfer, 1962; Hickey and Guillery, 1979). Here, we used high-resolution $\left(1.5 \times 1.5 \times 2 \mathrm{~mm}^{3}\right)$ functional magnetic resonance imaging (fMRI) at $3 \mathrm{~T}$ in humans to derive a detailed account of the retinotopic organization of the LGN, including estimates of the eccentricity magnification factor and of 
the visual field representation as a function of polar angle. In addition, we attempted to distinguish the $\mathrm{M}$ and $\mathrm{P}$ subdivisions of the LGN based on their different sensitivities to luminance contrast. Our results reveal a close correspondence in the topographic organization of the macaque and human LGN and support accounts of a constant magnification from the retina through the cortex.

\section{Materials and Methods}

Subjects. Seven subjects participated in the study, which was approved by the Institutional Review Panel of Princeton University. All of the subjects (22-30 years of age; three males, four females) were in good health with no history of psychiatric or neurological disorders and gave their informed written consent. Subjects had normal or corrected-to-normal visual acuity. All of the subjects participated in two separate scanning sessions to measure the polar angle and eccentricity components of the retinotopic maps in the LGN. Five of the subjects participated in an additional session to measure responses to varying stimulus contrast in the LGN. Visual cortex was scanned in four subjects for comparison purposes.

Visual display. The stimuli were generated on a Macintosh G4 computer (Apple Computer, Cupertino, CA) using MATLAB software (The MathWorks, Natick, MA) and Psychophysics Toolbox functions (Brainard, 1997; Pelli, 1997) and were projected from a PowerLite 7250 liquid crystal display projector (Epson, Long Beach, CA) outside the scanner room onto a translucent screen located at the end of the scanner bore. Subjects viewed the screen at a total path length of $60 \mathrm{~cm}$ through a mirror attached to the head coil. The screen subtended $30^{\circ}$ of visual angle in the horizontal dimension and $26^{\circ}$ in the vertical dimension. A trigger pulse from the scanner synchronized the start of the stimulus presentation to the beginning of the image acquisition.

Visual stimuli. Visual stimuli consisted of checkerboard patterns whose components reversed contrast at $8 \mathrm{~Hz}$. The full checkerboard pattern, of which only a portion was revealed at any point in time, encompassed the central $15^{\circ}$ of the visual field $\left(13^{\circ}\right.$ near the vertical meridian) and contained 24 radial sectors and 12 evenly spaced annuli (see Fig. 1). The luminances of the alternating bright and dark sections of the checkerboard were chosen such that the mean luminance of the stimulus was the same as that of the neutral gray background $\left(147.1 \mathrm{~cd} / \mathrm{m}^{2}\right)$; the contrast (defined as the difference in luminances divided by their sum) between the checkers was $97.9 \%$, except for the $10 \%$ condition described below. In all of the visual displays, a central fixation point was present, and subjects were instructed to maintain fixation for the duration of each scanning run while passively viewing the stimuli.

Rotating hemifield and expanding ring stimuli (see Fig. 1 $A, B$ ) were used to determine the polar angle and eccentricity components of the retinotopic maps in the LGN (DeYoe et al., 1994, 1996; Engel et al., 1994, 1997; Sereno et al., 1995). The visible portion of the rotating hemifield stimulus slowly and smoothly rotated counterclockwise about the point of fixation. The expanding ring stimulus consisted of an annulus with thickness equal to one-half of the radius of the visual display that expanded from the fixation point. The annulus increased in eccentricity (i.e., the distance from fixation) and wrapped around to the center once it reached the outer edge of the display. For two subjects, the ring stimulus was reversed; it contracted instead of expanding. Both the hemifield and ring stimuli swept through the visual field with a period of $32 \mathrm{sec}$, thereby evoking waves of activation in neurons through whose receptive fields they passed. Each region of the stimulated visual field was exposed to a flickering checkerboard pattern during one-half of the stimulus period and the neutral gray background during the other half. The stimulus waveform was thus a square wave whose temporal phase varied systematically throughout the visual field such that the position represented by an activated voxel could be inferred from the temporal phase of its evoked hemodynamic response.

An alternating hemifield stimulus (see Fig. 1C) was used to measure two points (10 and 100\%) of the contrast response function of the LGN. This stimulus comprised a flickering checkerboard pattern covering one hemifield but sparing the vertical meridian and central $1.1^{\circ}$ of the visual field. The pattern alternated between the left and right hemifields every $16 \mathrm{sec}$. In separate scanning runs, the stimulus was presented at either 100 or $10 \%$ contrast.

Data acquisition. Data were acquired with a 3 T Allegra head-dedicated MRI scanner (Siemens, Erlangen, Germany) using a standard birdcage head coil. Six to 14 series of 128 volumes each were acquired with 18 interleaved coronal slices ( $2 \mathrm{~mm}$ thick with no gap between slices, except for two subjects who were scanned using a $1 \mathrm{~mm}$ gap during the polar angle session) and a gradient echo, echo planar sequence with a 128 square matrix leading to an in-plane resolution of $1.5 \times 1.5 \mathrm{~mm}^{2}$ [repetition time (TR), 2 sec; echo time (TE), $41 \mathrm{msec}$; flip angle, $90^{\circ}$ ]. A partial Fourier factor of $7 / 8$ was used to acquire an asymmetric fraction of $\mathrm{k}$-space to reduce the acquisition time. The posterior edge of the acquisition volume was aligned in the midsagittal plane with the posterior edge of the corpus callosum to cover the posterior thalamus. Echo planar images were compared with a coaligned high-resolution anatomical scan of the same subject's brain taken at the beginning of the session (FLASH; TR, 150 msec; TE, 4.6 msec; flip angle, $90^{\circ}$; $256 \times 256$ matrix; six averages), and a high-resolution anatomical volume acquired in a separate session (MPRAGE sequence; TR, $2.5 \mathrm{sec}$; TE, $4.38 \mathrm{msec}$; flip angle, $8^{\circ}$; $256 \times 256$ matrix; $1 \mathrm{~mm}^{3}$ resolution).

The subjects' heads were surrounded by foam to reduce head movements; subject $\mathrm{S} 2$ also used a bite bar.

Data analysis. The first four volumes in each run were discarded. To compensate for subject head movement and scanner drift, the remaining volumes were registered (Woods et al., 1998) to the fifth volume obtained during the session. For each voxel in the volume, the linear trend in the fMRI time series was subtracted to remove any slow signal drift, typical in fMRI signals, and the time series was divided by its mean intensity, converting the data from the arbitrary intensity scale from the MRI scanner to units of percentage of signal modulation. The time series of each voxel were averaged across repeated scanning runs of the identical stimulus condition as follows: in the first session, six (in subjects S1, S2, S5, and S7) or 12 (in subjects S3, S4, and S6) runs for the rotating hemifield stimulus; in the second session, 12 runs for the expanding ring stimulus; and in the third session, six runs for the $100 \%$ contrast alternating hemifield stimulus and eight runs for the $10 \%$ contrast stimulus. The images obtained during the first cycle of visual stimulation $(32 \mathrm{sec})$ were discarded to avoid transient effects of signal saturation and to allow the hemodynamics to reach steady state. Therefore, the time series of each voxel contained 112 time points, representing seven cycles of visual stimulation.

A Fourier analysis was performed to identify voxels activated by the stimulus (Bandettini et al., 1993; Engel et al., 1997). For each voxel, the amplitude and phase (the temporal delay relative to the stimulus onset) of the harmonic at the stimulus frequency were determined by a Fourier transform of the mean time series of the voxel. The correlation coefficient $r$, between the harmonic and the time series, was computed as the amplitude of the harmonic component divided by the square root of the time series power. To correctly match the phase delay of the time series of each voxel to the phase of the stimulus, and thereby localize to the region of the visual field to which the underlying neurons responded best, the response phases were corrected for each subject's individual hemodynamic lag as measured in response to a $100 \%$ contrast alternating hemifield stimulus. For the purposes of comparison (but not quantitative analysis), the eccentricity maps (see Fig. 2) were anatomically registered (Jenkinson et al., 2002) for each subject to their polar angle maps. To report the Talairach coordinates (Talairach and Tournoux, 1988), the statistical maps and structural images for each subject were transformed into Talairach space using BrainVoyager software (Brain Innovation, Maastricht, The Netherlands).

Statistical maps were thresholded at $r \geq 0.25$, corresponding to an uncorrected $p<0.0038$. Regions of interest (ROIs) for each LGN were identified as contiguously activated voxel clusters in their anatomical locations, as determined from registered high-resolution structural images of each subject (O'Connor et al., 2002; Kastner et al., 2004). Care was taken to avoid including activations related to larger vascular structures into these ROIs.

The volumetric eccentricity magnification factor $(M)$ (Daniel and Whitteridge, 1961; Myerson et al., 1977) in the LGN is defined for each 


\section{A}
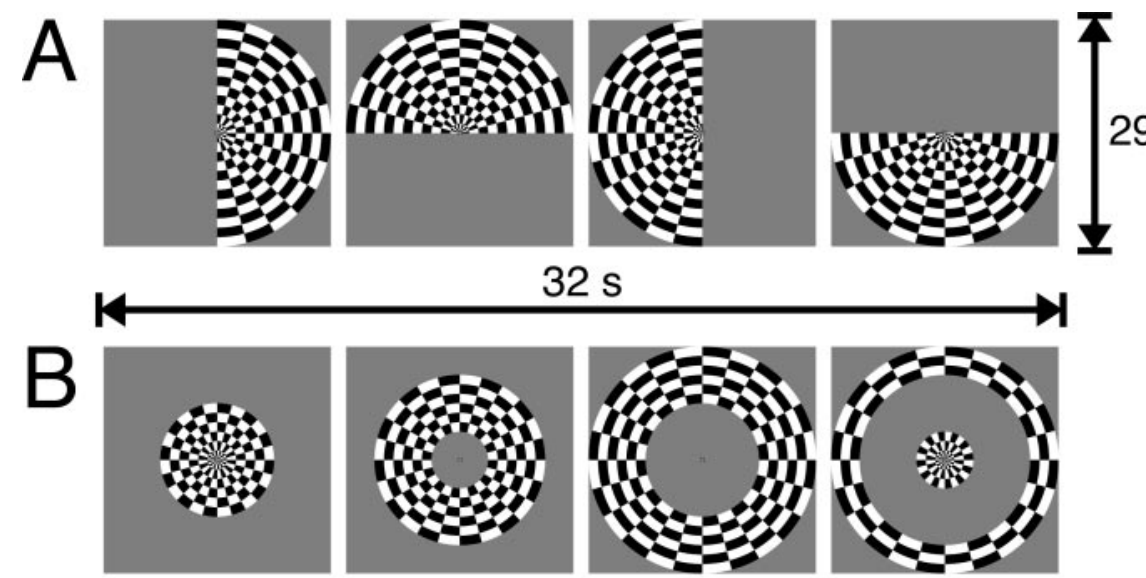

$32 \mathrm{~s}$
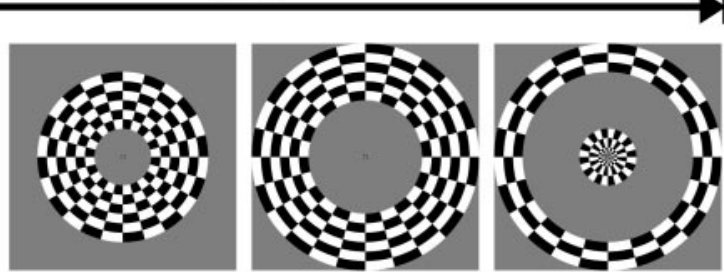

C

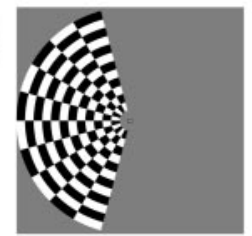

$16 \mathrm{~s}$

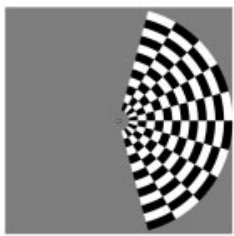

$16 \mathrm{~s}$

Figure 1. Visual stimuli. The stimuli were contrast-reversing checkerboard patterns. $A$, A hemifield pattern, smoothly rotating counterclockwise, was used to map the polar angle representation. $B$, An expanding or contracting ring pattern was used to map the representation of eccentricity. Four frames during the $32 \mathrm{sec}$ period are shown for the stimuli in $A$ and $B$. $C, A$ flickering checkerboard pattern that alternated every $16 \mathrm{sec}$ between the left and right hemifields was used to measure the response to stimulus contrast. In separate runs, the stimulus was presented at either 100 or $10 \%$ luminance contrast.

eccentricity in the visual field as the unit of LGN volume that represents a unit of visual field area:

$$
M(r) \equiv \frac{d V(r)}{d a(r)} \approx \pi r \frac{d V(r)}{d r},
$$

where $V(r)$ is the cumulative volume representing the visual hemifield at eccentricities $\leq r$, and the hemifield area $a(r)$ has been approximated as the planar projection. Conventionally (Schwartz, 1980; Van Essen et al., 1984), $M$ has been described with a power function of the form $M(r)=$ $A(r+B)^{-\mathrm{C}}$. For each of the seven subjects, the cumulative volume as a function of eccentricity (i.e., the LGN volume representing the area of the visual field from fixation to eccentricity $r$ ) was formed from the eccentricities determined for each voxel and the volume of all of the voxels representing that eccentricity or less. This function was then fit (nonlinear least squares) individually for each LGN in each subject to the following function:

$$
\begin{aligned}
V(r)=\pi A \int_{0}^{r-r_{0}} x(x+B)^{-C} d x \\
\quad=\frac{\pi A}{2-C}\left[\left(r-r_{0}-\frac{B}{1-C}\right)\left(r-r_{0}+B\right)^{1-C}+\frac{B^{2-C}}{1-C}\right],
\end{aligned}
$$

where $r_{0}$ is a correction factor indicating the minimum eccentricity of activation. The lack of activity evoked precisely at $0^{\circ}$ has been observed using similar procedures (Sereno et al., 1995) and may be attributable to an artifact of the periodic stimulus, a measurement resolution limitation, variance in the hemodynamic lag, or perhaps small fixational eye movements by the subjects. The mean parameters for the population were determined as those that best fit the average of the fitted functions for all of the subjects (aligned with $r_{0}=0$ ).

To compute volumetric variation as a function of polar angle, the visual field was divided into sixteen $22.5^{\circ}$ sectors, centered at $0^{\circ}$ (right horizontal meridian), $22.5^{\circ}, 45^{\circ}, 67.5^{\circ}, 90^{\circ}$ (top vertical meridian), $112.5^{\circ}, 135^{\circ}, 157.5^{\circ}, 180^{\circ}$ (left vertical meridian), $202.5^{\circ}, 225^{\circ}, 247.5^{\circ}$, $270^{\circ}$ (bottom vertical meridian), $292.5^{\circ}, 315^{\circ}$, and $337.5^{\circ}$. LGN voxels from each subject were sorted into these bins based on the phase of their responses. The bins centered on the top and bottom vertical meridians contained voxels from both the left and right LGN. The volumes of voxels within each sector were totaled and averaged across subjects, and the mean polar angle representation was calculated as the volume in each sector divided by the area of the sector. The two subjects who were scanned during the polar angle session with a $1 \mathrm{~mm}$ gap between slices were excluded from this analysis. To measure the extent to which partial volume effects may have influenced the results, we repeated this analysis on the layer of subthreshold voxels immediately adjacent to each LGN ROI, separating the voxels from each left and right LGN and subtracting the isotropic noise.

In an attempt to distinguish the $\mathrm{M}$ and $\mathrm{P}$ subdivisions of the LGN, we defined three criteria that should be fulfilled by voxels containing significant fractions of $\mathrm{M}$ neurons. The first criterion was high contrast sensitivity. We expected that voxels containing $\mathrm{M}$ neurons would be responsive to low stimulus contrast and should therefore be strongly activated by the $10 \%$ contrast stimulus. We defined a candidate ROI containing the voxels exhibiting a significant ( $r \geq 0.25$ ) response to the $10 \%$ contrast stimulus and tested these voxels on the remaining two criteria. The second criterion was weak contrast modulation. In the macaque, most $\mathrm{P}$ neurons exhibit near-baseline responses for $10 \%$ contrast stimuli, whereas most $\mathrm{M}$ neurons respond to $10 \%$ contrast stimuli at or above their half-maximum response (Sclar et al., 1990). The contrast sensitivities of $\mathrm{M}$ and $\mathrm{P}$ cells in the human LGN have not yet been studied, but the contrast sensitivity of $M$ cells can be indirectly estimated from the population response of the middle temporal (MT) cortical area, which appears to be dominated by inputs from $\mathrm{M}$ cells (Maunsell et al., 1990). In previous fMRI studies, area MT has exhibited reliable responses to stimulus contrasts as low as 1.6\% (Tootell et al., 1995) and response saturation at stimulus contrasts as low as 4\% (Kastner et al., 2004). Voxels containing mostly $\mathrm{P}$ neurons should exhibit substantial modulation of their responses between the 10 and 100\% contrast stimuli, whereas voxels containing mostly $M$ neurons should exhibit relatively modest modulations in this contrast range. To quantify the contrast modulation, we defined a contrast modulation index (CMI) based on the response amplitudes to the $100 \%\left(A_{100 \%}\right)$ and $10 \%\left(A_{10 \%}\right)$ contrast stimuli: $\mathrm{CMI} \equiv\left(A_{100 \%}-A_{10 \%}\right) /\left(A_{100 \%}+A_{10 \%}\right)$. We examined the third criterion, anatomical location, for those voxels with $\mathrm{CMI}<0.25$. The $\mathrm{M}$ layers in humans are expected to be most prominent on the inferior, posterior, and medial surfaces of the LGN (Kupfer, 1962; Hickey and Guillery, 1979).

To further examine the responses of subsets of the LGN voxels, including ROIs representing voxels unresponsive to the $10 \%$ contrast stimulus, and voxels responsive to the $10 \%$ contrast stimulus exhibiting either small $(\mathrm{CMI}<0.25)$ or large $(\mathrm{CMI} \geq 0.25)$ contrast response modulation, we computed the average time series response for each condition (see Fig. 8), first averaging over voxels within each ROI in each LGN of each subject and then averaging together these mean time series across subjects for the left and right LGN individually.

\section{Results}

\section{Polar angle maps}

The polar angle component of the retinotopic map in the LGN was measured using a smoothly rotating, flickering hemifield checkerboard stimulus (Fig. 1A). Bilateral activation was observed in all seven of the subjects, and the activations of each LGN 
were strictly confined to stimulation of the contralateral hemifield. Across subjects, the mean volume of each LGN $(n=14)$ activated by the rotating hemifield stimulus was $440 \pm 25 \mathrm{~mm}^{3}$ (mean \pm SEM). The volumes of the left and right LGN in each subject were significantly correlated $(r=0.75 ; p=0.051)$. The activations typically extended through five slices and were contained entirely within $1 \mathrm{~cm}^{3}$. The mean Talairach coordinates for centers of mass of the activations were $+23,-23$, and -3 for the right LGN and $-21,-23$, and -3 for the left LGN, similar to those obtained in previous studies (O'Connor et al., 2002; Kastner et al., 2004).

Individual activation maps are shown for two representative subjects (S1 and S2) in Figure 2. Activated voxels whose fMRI time series were correlated with the fundamental frequency of the stimulus, $r \geq 0.25$, are shown in the left and right columns. The activation maps, overlaid on structural scans (shown in the middle panel), are displayed in $15 \times 12 \mathrm{~mm}^{2}$ windows for five contiguous slices. The color of each voxel was determined by the phase of its response and indicates the region of the visual field to which it was most responsive, as depicted in the color legend at the top of each column. Regions of the top visual field are indicated in red-yellow; regions along the horizontal meridian are indicated in green; and regions of the bottom visual field are indicated in blue. In the coronal plane, the representation of the horizontal meridian was oriented at an $\sim 45^{\circ}$ angle, dividing the bottom visual field, represented in the medial-superior section of the LGN, and the top visual field, represented in the lateral-inferior section. Although the extent of activations varied somewhat among subjects, the general pattern of retinotopic polar angle organization was consistent.

Time series of fMRI signals from three adjacent voxels of the left LGN of one subject (S1) are shown in Figure $3 A$. The temporally smoothed time series were averaged over six scanning runs from the same session and illustrate the fMRI signals evoked by the checkerboard stimulus. The stimulus passed seven times through the region of the visual field represented by each voxel and thereby evoked seven peaks of activity. Although the voxels were small and adjacent, their response phases could be reliably distinguished as representing separate regions of the visual field: the bottom field (blue voxel), the horizontal meridian (green voxel), and the top field (red voxel). For comparison, time series of three identically sized single voxels from visual cortex with similar phase responses are shown in Figure $3 B$ for the same subject. The mean amplitude (mean to peak) of the responses in the three LGN voxels shown was $1.65 \%$, compared with a mean amplitude of $3.92 \%$ for the cortical voxels; similar response ratios between LGN and visual cortex were reported in previous studies using a lower spatial resolution (O'Connor et al., 2002; Kastner et al., 2004).

\section{Polar angle representation}

The polar angle representation of the LGN was computed from the mean LGN volume representing a square degree of visual angle within each of 16 separate sectors of the visual field, as shown in Figure 4. This analysis revealed that the horizontal meridian was significantly overrepresented relative to the vertical meridian. The mean volume in each LGN $(n=10)$, representing the $22.5^{\circ}$ sector centered on the horizontal meridian, was $84.2 \pm$ $9.1 \mathrm{~mm}^{3}$, significantly greater than the mean volume representing the two $11.25^{\circ}$ sectors abutting the vertical meridian, $14.0 \pm 2.9$ $\mathrm{mm}^{3}$ (paired two-tailed $t$ test; $t_{(9)}=7.86 ; p=0.000025$ ). The bottom visual field representation was slightly larger than the top visual field representation, $230 \pm 24$ versus $207 \pm 22 \mathrm{~mm}^{3}$, but this difference was not statistically significant $\left(t_{(9)}=0.83 ; p=0.43\right)$.

One possible explanation for the observed anisotropy could be a selective undersampling of the vertical meridian as a result of partial volume effects (Haacke et al., 1994; Logothetis et al., 2002). In a coronal section of the LGN, the horizontal meridian runs through the center of the structure, whereas the vertical meridian is represented along the edges in which partial volumes could confound the number of activated voxels. To investigate this possibility, we analyzed the phase angles in the layer of voxels immediately surrounding the LGN. The signal from these voxels had not individually reached our criterion for statistical significance, and they were not included in the LGN ROIs. A latent 


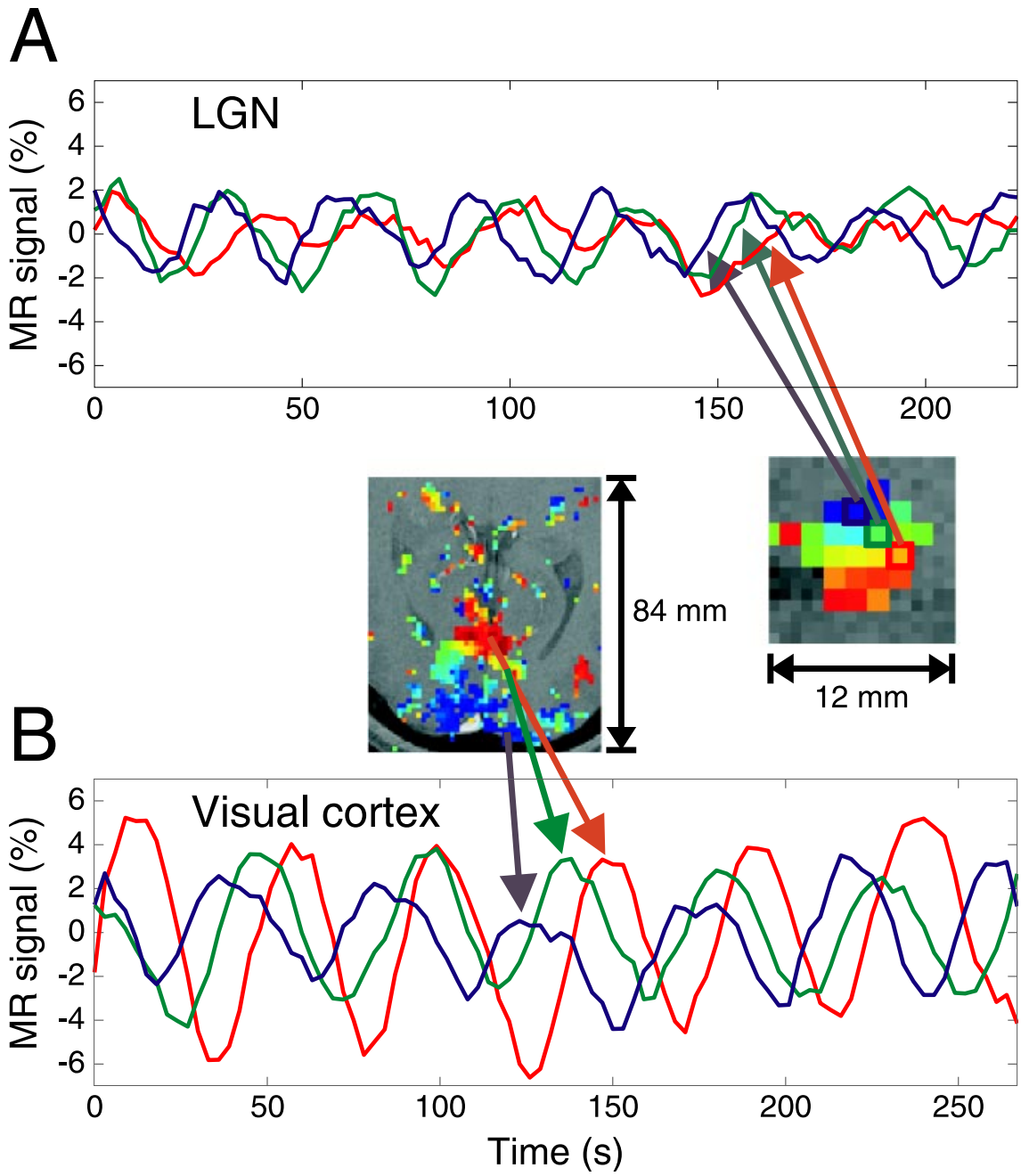

Figure 3. Time series of $\mathrm{fMRI}$ signals in the LGN and V1. A, Time series of $\mathrm{fMRI}$ signals are shown from three individual voxels within the LGN region activated by the rotating hemifield stimulus in one representative subject (S1). The voxels were adjacent (see arrows), as shown in the magnified view of the left LGN (the same color code and format as in Fig. 2). The time series were averaged over six scanning runs and have been temporally smoothed by averaging five sequential points. Although the small voxels are adjacent, their time series exhibited distinct response phases, allowing the voxels to be reliably assigned to different regions of the visual field. $B$, Smoothed fMRI time series, averaged over five scanning runs, are shown for comparison from three separate voxels in visual cortex of the same subject. The scanning parameters for these cortical data were the same as for the LGN, with identical voxel resolution but with TR $=3 \mathrm{sec}$. Each run contained 105 volumes of 28 interleaved axial slices, and the rotating hemifield stimulus had seven cycles and a period of $45 \mathrm{sec}$, of which only the last six cycles are shown.

signal might still be detectable at the population level, however, and we examined the distribution of responses in the group of voxels to investigate any systematic biases in the response phases. After subtraction of an estimate of the isotropic noise, the phase distribution of these voxels appeared similar to that of the LGN voxels; the best fitting ellipse to this distribution had a ratio of the major to minor axes of 2.88, strongly biased in favor of the horizontal meridian representation. Given that both the polar angle representation of the LGN itself and the set of voxels immediately surrounding the LGN favored the horizontal meridian, partial volume effects may have contributed but are unlikely to fully explain the asymmetric representations of the horizontal and vertical meridians in the human LGN.

\section{Eccentricity maps}

The eccentricity maps of the central $15^{\circ}$ of the visual field were measured in response to an expanding or contracting flickering checkerboard ring stimulus (Fig. $1 B$ ). These stimuli activated the
LGN bilaterally in all seven of the subjects. Across subjects, the mean activated volume for each LGN $(n=14)$ was $197 \pm 11$ $\mathrm{mm}^{3}$, significantly smaller than that activated by the rotating hemifield stimulus $\left(t_{(13)}=8.65 ; p=9.3 \times 10^{-7}\right)$, perhaps reflecting the lower effectiveness of the expanding ring stimulus that has also been observed in cortical areas. Within each subject, the activated volumes of the left and right LGN were significantly correlated ( $r=0.88$; $p=0.0095)$, but there was no significant correlation between the volumes activated by the expanding ring and rotating hemifield stimuli $(r=-0.028$; $p=0.92)$.

Eccentricity maps are shown for the same subjects and in register with the polar angle retinotopic maps in Figure 2 (far columns on the right and left). The color code, as indicated by the legend at the top of each column, indicates the region of the visual field to which each voxel was most responsive. Voxels representing the central $5^{\circ}$ are indicated in dark to light blue; those representing $5-10^{\circ}$ are indicated in cyan to green to yellow; and those representing the peripheral $10-15^{\circ}$ are indicated in orange to red. The central $5^{\circ}$ were represented mainly in the posterior portion of the LGN; in more anterior planes, the representation of the central $5^{\circ}$ was confined to superior sections. More peripheral representations of the visual field were systematically arranged in anterior and inferior regions of the nucleus. As with the polar angle maps, the organization of the eccentricity maps was consistent across subjects.

\section{Eccentricity magnification factor}

The magnification factor of the LGN was computed based on the volumetric eccentricity data. The magnification factor has typically been described with a power function of the form $M(r)=A(r+B)^{-\mathrm{C}}$, where $r$ is the eccentricity (in degrees) and the exponent $C$ is often fixed to 1 or 2 for linear or areal magnification factors, respectively (Schwartz, 1980; Van Essen et al., 1984). To determine the parameters of this function for our data, we fit its integral over the area of the visual field to the cumulative volume of the LGN for each subject. Examples for three representative subjects are shown in Figure 5 (see Materials and Methods for details). No differences were apparent between subjects who viewed expanding (S1-S5) or contracting (S6 and S7) ring stimuli. The cumulative volumes were similarly shaped among the subjects, although differing in slope and extent. For many of the subjects, the cumulative volume function was steep and nearly linear for the initial $2-5^{\circ}$ of eccentricity, after which the slope abruptly became shallower. A similar broken function has been observed in macaque visual cortex, in which the receptive field sizes are nearly constant within the foveal $5^{\circ}$ and begin to increase rapidly thereafter (Van Essen et al., 1984). 


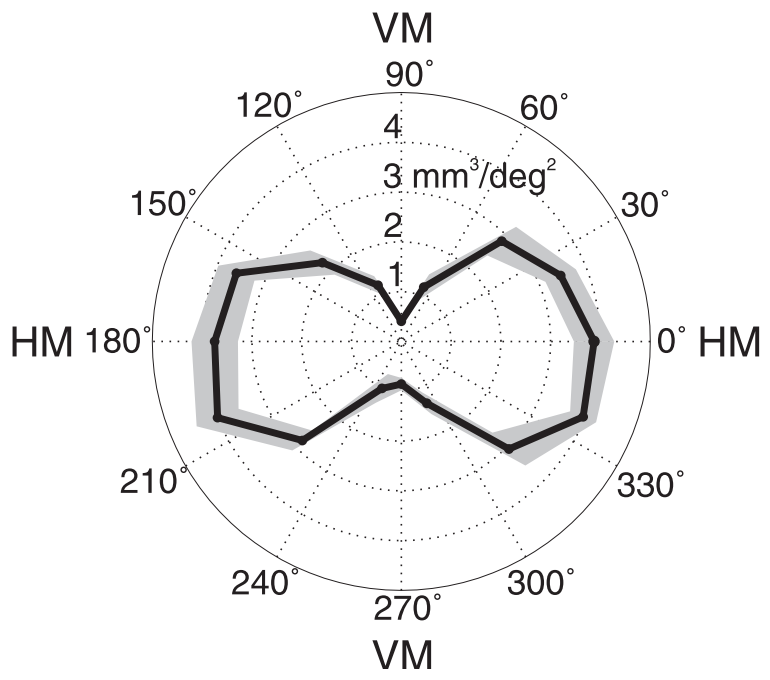

Figure 4. Polar angle representation in the $L G N$. The representations in the $L G N$ of the visual field at different polar angles were measured for the central $15^{\circ}$ of eccentricity by dividing the visual field into 16 sectors and sorting voxels activated by the rotating hemifield stimulus according to their phase responses into each sector. Results for the left and right $L G N$ are shown combined in one polar plot. The points and lines trace the mean volume per unit area of the visual field for each of the sectors, averaged over the subjects $(n=5)$. The gray regions indicate the extent of the SEM. Significantly more volume of the LGN represents the horizontal meridian (HM) than the vertical meridian (VM).

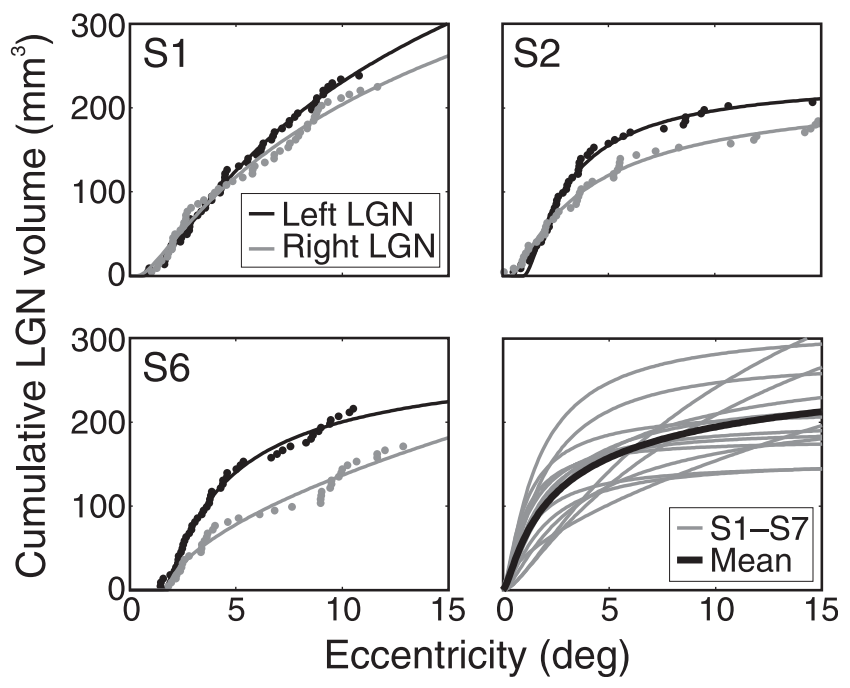

Figure 5. Eccentricity magnification factors in the LGN. Eccentricity magnification factors for three representative subjects $(S 1, S 2, S 6)$ in each $L G N$ are shown, computed based on the phase responses of voxels activated by the expanding ring stimulus. The cumulative volume representing the area of the visual field from the fixation point to an eccentricity of $\leq r$ was fit to the integral of the magnification function $M(r)=A(r+B)^{-C}$ over the area of the visual hemifield (see Materials and Methods). The fits for each LGN in each of the seven subjects are superimposed in the bottom right panel, along with the mean cumulative volume function fit for all of the subjects, which was $M(r)=46.6(r+0.52)^{-2.43}$.

An estimate of the eccentricity magnification function for the population was derived by fitting the average of the individual means (Fig. 5, bottom right), yielding $M(r)=46.6(r+0.52)^{-2.43}$ $\mathrm{mm}^{3} / \mathrm{o}^{2}$. These parameters were compared with previous results from macaque LGN and macaque and human V1 (see supplementary material, available at www.jneurosci.org), and the relative cumulative volume functions are plotted in Figure 6. The volumetric (for LGN in millimeters cubed per degree squared) and areal (for V1 in millimeters squared per degree squared)

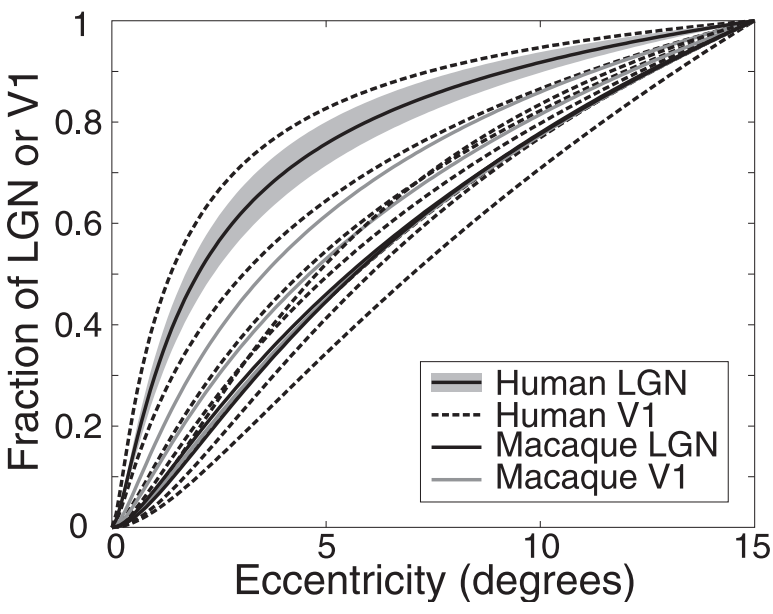

Figure 6. Cumulative volume functions for the human and macaque LGN and V1. The cumulative volumes (for LGN) or areas (for V1) as a function of eccentricity are plotted as a fraction of the volume or area at $15^{\circ}$, based on the reports of the magnification factor in the literature. The $15^{\circ}$ scaling is necessary to compare the different structures and species. The solid black line surrounded by the gray shaded area represents the mean measurements from the subjects in the present study, with the shaded area representing the extent of the SEM. The dashed black lines represent measurements in human V1, from studies using techniques including measurement of the position of phosphenes evoked by migraines (Grüsser, 1995), electrical stimulation of the cortex (Brindley and Lewin, 1968; Cowey and Rolls, 1974), evoked potentials (Slotnick et al., 2001), and fMRI (Engel et al., 1994, 1997; Sereno et al., 1995; Dougherty et al., 2003; Duncan and Boynton, 2003). Analyses of the macaque LGN (solid black lines) and V1 (solid gray lines) were based on single-cell physiology (Hubel and Wiesel, 1974; Malpeli and Baker, 1975; Hubel and Freeman, 1977; Connolly and Van Essen, 1984; Van Essen et al., 1984; Malpeli et al., 1996) and deoxyglucose autoradiography (Tootell et al., 1982, 1988). For details of the parameters of these studies, see supplementary material, available at www.jneurosci.org.

magnification factors both indicate the relative numbers of neurons devoted to each region of the visual field and can be directly compared (Myerson et al., 1977), because the thickness and density of V1 are relatively uniform. Our measurements for the LGN are similar to those obtained for human V1 and are within the variance obtained with the different methods, such as visually evoked potentials, fMRI, and phosphenes evoked by migraines or electrical stimulation. The function for the human LGN indicates a relative overrepresentation of the fovea compared with macaque LGN.

\section{Contrast sensitivity maps}

In an attempt to dissociate the $\mathrm{M}$ and $\mathrm{P}$ subdivisions of the LGN, two points of the contrast sensitivity function in five subjects were measured using a flickering checkerboard stimulus with 10 or $100 \%$ luminance contrast that alternated between the left and right hemifields (Fig. 1C). Based on the anatomy (Hickey and Guillery, 1979) and the different sensitivities to stimulus contrast of the two populations of neurons (Derrington and Lennie, 1984; Sclar et al., 1990), we assumed that the M subdivision could be identified by three criteria: high sensitivity to low stimulus contrast, small or no differences in responses to 10 and $100 \%$ contrast stimuli, and anatomical location.

Based on the anatomy, we expected M portions of the LGN to be located medially, inferiorly, and posteriorly. Typically, the M layers are flat and located on the inferior surface of the LGN, but particularly in the posterior planes, the LGN is oriented at an angle such that the $\mathrm{M}$ layers are located medially. Furthermore, the LGN often exhibits folding such that the M layers would be located in the interior of the structure. Across individuals, the LGN exhibits considerable variation in its size as well as its lam- 
A
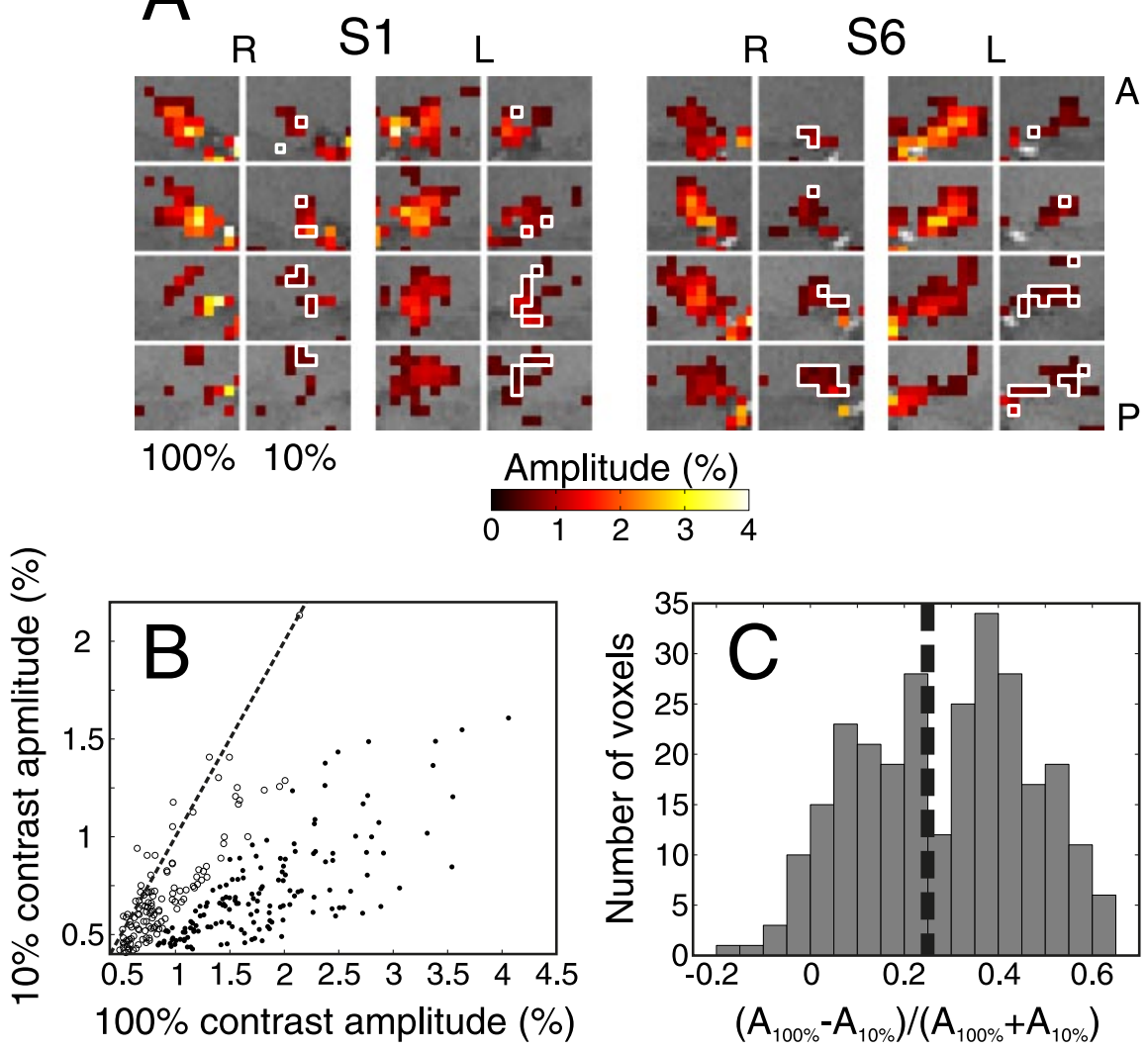

Figure 7. Contrast sensitivity maps. A, Activity evoked by the alternating hemifield stimulus in the anatomical location of the left $(L)$ and right $(R) L G N$ is shown for two representative subjects. Four sequential slices are shown, ordered anterior $(A)$ to posterior $(P)$. The left column of each pair indicates the amplitude of the response to the $100 \%$ contrast stimulus, and the right column indicates the response to the $10 \%$ contrast stimulus. Only those voxels with correlations to the fundamental frequency of the stimulus, $r \geq 0.25$, are shown. Voxels surrounded by white lines responded similarly to the 10 and $100 \%$ contrast stimuli (see Results and $B$ ). Based on their high contrast sensitivity, these voxels are likely dominated by M neurons. $B$, For each subject ( $n=$ 5) and each voxel activated by both the 10 and 100\% contrast stimuli ( $r \geq 0.25)$, the amplitudes of the mean fMRI time series, evoked by the $100 \%\left(A_{100 \%}\right)$ and $10 \%\left(A_{10 \%}\right)$ contrast alternating hemifield stimuli, are plotted against each other. The dashed diagonal line indicates equality between the amplitudes. The unfilled circles represent the voxels whose CMls, defined as $\left(A_{100 \%}-A_{10 \%}\right) /\left(A_{100 \%}+A_{10 \%}\right)$, were $<0.25$. These voxels are bordered with solid white lines in $A$. $C$, The distribution of $C M I$. Voxels predominately containing $M$ neurons are expected to be similarly activated by both the 10 and $100 \%$ contrast stimuli, and hence have a small CMI. P voxels are expected to have a strong differential response to the 10 and $100 \%$ contrast stimuli and therefore will have a large CMI. The dotted vertical line marks the 0.25 threshold used to select the voxels in $A$ and $B$.

tions in the right and left LGN evoked by the 10 and $100 \%$ contrast stimuli are shown, similar to the format used in Figure 2. Four sequential slices are displayed for each LGN, and in each pair of columns, the voxels are shown whose fMRI signals were correlated with the fundamental frequency of the visual stimulus, $r \geq 0.25$. Activations evoked by the $100 \%$ contrast stimulus are shown in the left column of each pair.

Subsequently, we identified the regions within this ROI that were most responsive to the $10 \%$ contrast stimulus and therefore candidate areas to contain the $\mathrm{M}$ subdivision. The voxels activated by the $10 \%$ contrast stimulus, shown in the right column of each pair in Figure $7 A$, constituted a subset of the voxels activated by the $100 \%$ contrast stimulus. The voxels most responsive to the $10 \%$ contrast stimulus formed clusters that varied among the subjects in location relative to the activations evoked by the $100 \%$ contrast stimulus.

The second criterion that we used to identify the $M$ subdivisions was that the responses of $\mathrm{M}$ voxels evoked by the $10 \%$ contrast stimulus should be nearly saturated and marginally different from the responses evoked by the $100 \%$ contrast stimulus, whereas $\mathrm{P}$ voxels should exhibit larger differences in response to the two contrast stimuli. Therefore, we analyzed the contrast modulation for those voxels that were reliably $(r \geq 0.25)$ activated by both the 10 and $100 \%$ contrast stimuli. For these voxels, the averaged response amplitudes evoked by the 10 and $100 \%$ contrast stimuli are compared in Figure $7 B$. A high correlation is evident, such that for each voxel, the larger the amplitude evoked by the $100 \%$ contrast stimulus, the larger the amplitude tended to be evoked by the $10 \%$ contrast stimulus $(r=0.59 ; p=7.3 \times$

inar organization, more so than in other species (Hickey and Guillery, 1979; Andrews et al., 1997). As a result of this variability, in some subjects, the $\mathrm{M}$ layers might be oriented in positions that make their discrimination difficult from the P layers at the resolution used in this study.

In our analysis, we assumed that the $100 \%$ contrast stimulus activated both the $\mathrm{P}$ and $\mathrm{M}$ sections of the LGN, and its evoked activity was used to define an ROI encompassing both. The mean volume for each LGN $(n=10)$ activated by this stimulus, which spared the central $1.1^{\circ}$ and vertical meridian, was $326 \pm 36 \mathrm{~mm}^{3}$, marginally smaller than the volumes activated by the rotating hemifield stimulus $\left(t_{(9)}=2.17 ; p=0.059\right)$ and significantly greater than those activated by the expanding ring stimulus $\left(t_{(9)}=3.22 ; p=0.011\right)$. The left and right LGN volumes within each subject were significantly correlated $(r=0.89 ; p=0.046)$, but the volumes were not correlated with those activated by the rotating hemifield $(r=-0.023 ; p=0.95)$ or expanding ring stimuli $(r=0.23 ; p=0.53)$. Activation maps from two representative subjects ( 1 and $\mathrm{S} 6$ ) are shown in Figure 7A. The activa- $\left.10^{-27}\right)$. The linear regression line has a slope of 0.22 , but the population is distributed, including voxels clustered around the unity slope line, which indicates equality in the amplitudes evoked by the two contrast stimuli (Fig. 7B). To quantify the response modulation, we calculated a CMI for each voxel, defined as $\left(A_{100 \%}-A_{10 \%}\right) /\left(A_{100 \%}+A_{10 \%}\right)$, where $A_{100 \%}$ and $A_{10 \%}$ are the response amplitudes evoked by the 100 and $10 \%$ contrast stimuli, respectively. Voxels with CMI values near 0 were weakly modulated by the increase from 10 to $100 \%$ stimulus contrast, and those voxels with CMI near 1 were strongly modulated. The distribution of the CMIs is shown in Figure 7C. The 44.3\% (121 of 273) of voxels with CMIs $<0.25$ are indicated by unfilled circles in Figure $7 B$ and are bordered with white lines in Figure $7 A$. Therefore, of the voxels contained within the LGN ROI (defined as voxels activated, $r \geq 0.25$, by the $100 \%$ contrast stimulus), $16.7 \%$ (121 of 725 ) fulfilled both criteria, exhibiting significant responses to the $10 \%$ contrast stimulus and exhibiting contrast saturation $(\mathrm{CMI}<0.25)$. These are the most likely candidates for voxels dominated by $\mathrm{M}$ responses. Although the anatomical lo- 

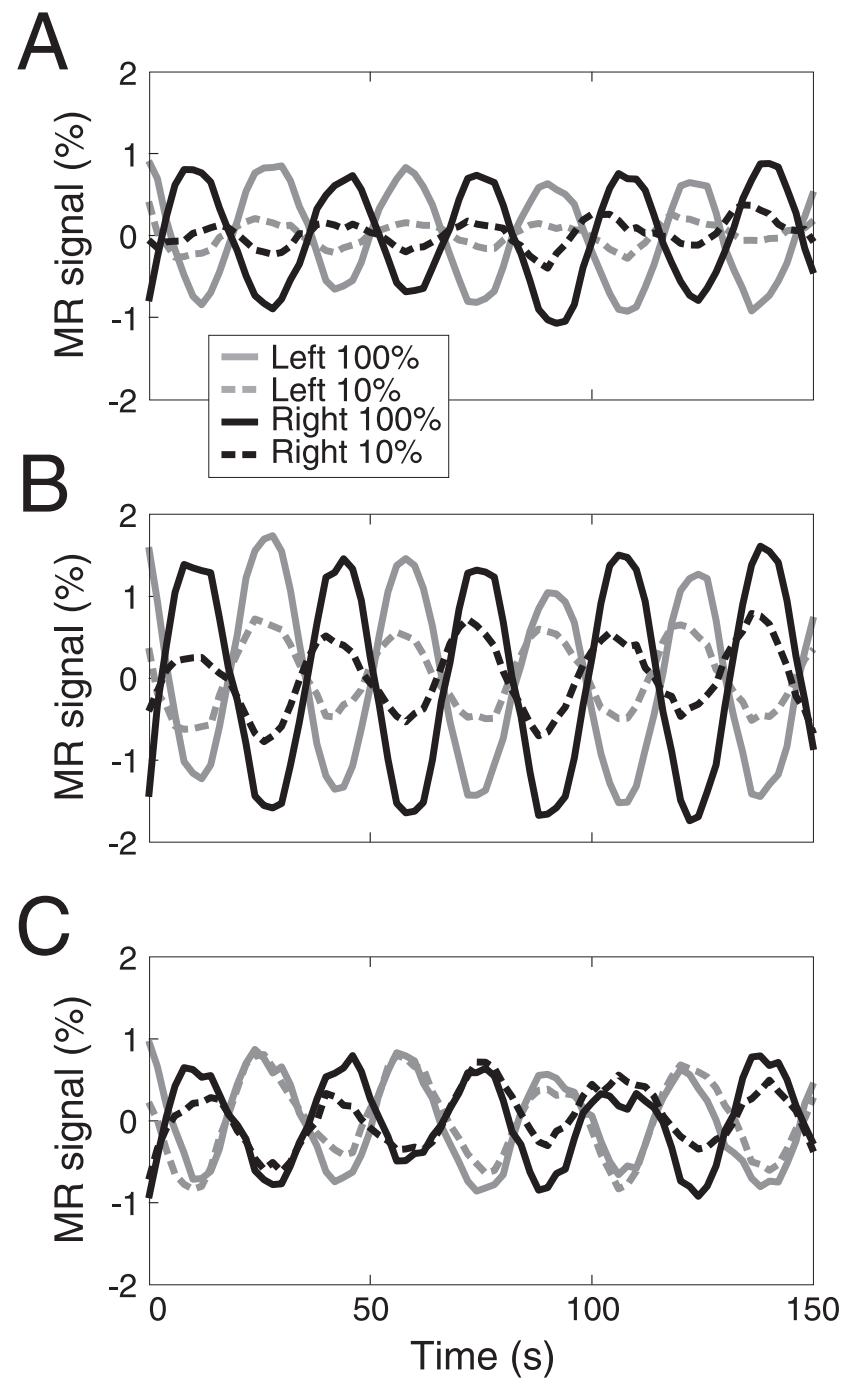

Figure 8. Time series of fMRI signals in the LGN. The responses for three subsets of voxels within the left and right LGN are shown for the 100 and $10 \%$ contrast stimulus. The time series were averaged over the selected voxels within the LGN for each subject and over six to eight scanning runs, and then averaged over five subjects $(\mathrm{S} 1-\mathrm{S4}, \mathrm{S6})$ and temporally smoothed. Only 150 sec of each time series are shown. $A$, Voxels responsive to the $100 \%$ contrast stimulus ( $r \geq$ $0.25)$ but unresponsive to the $10 \%$ contrast stimulus $(r<0.25)$. B, Voxels responsive to the $10 \%$ contrast stimulus ( $r \geq 0.25$ ) and exhibiting large differences in response amplitudes to the $10 \%$ $\left(A_{10 \%}\right)$ and $100 \%\left(A_{100 \%}\right)$ contrast stimuli, with CMls as follows: $\left(A_{100 \%}-A_{10 \%}\right) /\left(A_{100 \%}+\right.$ $\left.A_{10 \%}\right) \geq 0.25$. C, Voxels responsive to the $10 \%$ contrast stimulus $(r \geq 0.25)$ exhibiting small response differences for the 10 and $100 \%$ contrast stimuli, $\mathrm{CMI}<0.25$.

cations of these voxels varied, when clustered, they tended to be located medially and/or posteriorly, as is the case for the two subjects shown in Figure $7 A$.

To substantiate our analysis further, we evaluated the fMRI time series for three subsets of LGN voxels. Figure $8 \mathrm{~A}$ shows the average time series for those voxels that were activated by the $100 \%$ but not by the $10 \%$ contrast stimulus. Figure $8 B$ shows the average time series for those voxels that were activated by both the 10 and $100 \%$ stimuli but had CMIs $\geq 0.25$. The large contrast modulation evident in these time series may indicate that these signals originated primarily from $\mathrm{P}$ neurons. Figure $8 \mathrm{C}$ shows the time series for those voxels that were activated by the $10 \%$ contrast stimulus and had CMIs $<0.25$, indicating small differences in the responses to the 10 and $100 \%$ contrast stimuli, as is evident in the time course of the fMRI signals. These voxels may contain significant numbers of $\mathrm{M}$ neurons and are indicated by white outlines in Figure $7 A$ and unfilled circles in Figure $7 B$. It is evident from the time series and the scatter plot in Figure $7 B$ that the weakly modulated voxels tended to be those with the smallest dynamic range, exhibiting the smallest amplitudes in response to the $100 \%$ contrast stimulus.

\section{Discussion}

Using high-resolution fMRI at $3 \mathrm{~T}$, we measured the polar angle and eccentricity components of the retinotopic map in the human LGN. The retinotopy was consistent among subjects and was sufficiently detailed to allow quantifications of the eccentricity magnification factor and the polar angle representation. We were also able to distinguish functional subdivisions within the LGN based on differences in sensitivity to luminance contrast that may relate to the $\mathrm{M}$ and $\mathrm{P}$ subdivisions of the LGN.

\section{High-resolution fMRI of the LGN}

It has been difficult to study subcortical nuclei in the human brain using fMRI because of their small sizes and deep locations. Typical fMRI experiments that are conducted with voxel sizes of $\geq(3 \mathrm{~mm})^{3}$ cannot reveal detailed topographic maps of brain structures measuring less than $1 \mathrm{~cm}^{3}$, such as the LGN. The use of smaller voxel sizes, on the other hand, reduces the signal-to-noise ratio (SNR), which is proportional to the voxel volume. We obtained robust signals in the present study using a voxel size of $1.5 \times 1.5 \times 2 \mathrm{~mm}^{3}$. This may be attributable to a reduction in partial volume effects, which normally occur when some fraction of a voxel volume is positioned over inactive tissue (Haacke et al., 1994; Logothetis et al., 2002).

Notably, we observed distinct phase responses in adjacent voxels in the LGN. In area V1 of the human visual cortex, the resolution of $\mathrm{fMRI}$ has been estimated to have a line spread function with a full width at half-maximum (FWHM) of $3.5 \mathrm{~mm}$ (Engel et al., 1997), which is primarily determined by horizontal connections (Das and Gilbert, 1995; Menon and Goodyear, 1999). In the LGN, the resolution is not limited by feedforward horizontal connections, which may explain why we were able to resolve topographic details from adjacent voxels of small size. Interestingly, the point spread function of the hemodynamic response has been estimated in the cat LGN to have an FWHM of $1.6 \mathrm{~mm}$ (Thompson et al., 2003), closely matching the voxel size used in the present study.

Topographic organization of the LGN in macaque and human The topographic organization of the LGN has been studied extensively in macaques, using anatomical (Brouwer and Zeemann, 1926), physiological (Kaas et al., 1972; Malpeli and Baker, 1975; Connolly and Van Essen, 1984; Malpeli et al., 1996; Erwin et al., 1999), and lesion (Clark and Penman, 1934) techniques. The functional topography of the human LGN found in the present study has striking similarities with that reported in the macaque (see Introduction). We were not able to resolve individual layers of the LGN, but because the layers are spatially registered (Kaas et al., 1972, 1978), we were able to investigate the general topographical organization of the structure. Our measurements confirm and extend what is known about the human LGN by providing detail of its topographic organization and quantitative measures of magnification factors for polar angle and eccentricity. Until recently, the topography of the human LGN could only be studied through comparisons of clinical visual defects and cortical or retinal lesions to LGN degeneration observed postmortem (Rönne, 1910, 1913, 1914; Mackenzie, 1934; Juba and 
Szatmári, 1937; Kupfer, 1962; Hickey and Guillery, 1979). These studies agree that the upper and lower visual fields are represented inferiorly and superiorly, which has also been confirmed using fMRI (Chen et al., 1999), but they disagree on other issues. The foveal regions of the visual field are represented in the posterior part of the nucleus, but the extent of that representation is quite unclear. Kupfer (1962) found that the portion of the LGN representing the macula encompassed the posterior two-thirds to three-fourths of the LGN volume, whereas Hickey and Guillery (1979) suggested that the central $15^{\circ}$ occupied approximately one-half of the volume. The present study only examined the central $15^{\circ}$ of the visual field, but if the magnification function that we measured can be assumed to extend smoothly and continuously into the periphery, our results suggest that the central $15^{\circ}$ of the visual field occupies $79.5 \%$ of the total LGN volume (the volume extrapolated at $90^{\circ}$ ).

\section{Eccentricity magnification factor}

The eccentricity magnification factor $M$ has been measured using a number of techniques in both macaque and human V1 and in the macaque LGN (for details, see supplementary material, available at www.jneurosci.org). The measurements are all consistent with an inverse-square relationship between the magnification factor and eccentricity, although there is considerable variability among the exact functional parameters, even among studies using similar techniques. Our results show a greater representation of the fovea than in the macaque LGN or V1 and are within the range of those obtained in human V1. Several studies have suggested that $\mathrm{V} 1$ in primates exhibits an additional magnification of the foveal representations relative to the LGN (Malpeli and Baker, 1975; Myerson et al., 1977; Connolly and Van Essen, 1984; Van Essen et al., 1984; Perry and Cowey, 1985; Azzopardi and Cowey, 1996). However, a comparison of our measurements with those of V1 from the literature shows no such obvious difference; therefore, our results suggest that the magnification factor is preserved from the retina through the cortex, in agreement with other studies (Webb and Kaas, 1976; Schein and de Monasterio, 1987; Wässle et al., 1989, 1990; Malpeli et al., 1996).

\section{Polar angle representation}

In addition to the eccentricity magnification factor, we also measured the representation of the visual field as a function of polar angle. Our estimates indicate that the volume of the LGN representing the horizontal meridian is considerably larger than that devoted to the vertical meridian. In human and macaque retinas, the distribution of ganglion cells is significantly anisotropic, elongated along the horizontal meridian into a modest visual streak (Stone and Johnston, 1981; Perry and Cowey, 1985; Curcio and Allen, 1990; Curcio et al., 1990). Functionally, peripheral visual acuity is enhanced along the visual streak (Anderson et al., 1992). In the macaque LGN, an overrepresentation of the horizontal relative to the vertical meridian has been noted in all of the $\mathrm{P}$ layers (Connolly and Van Essen, 1984). Our observed differences were substantially larger than those found in the macaque LGN; however, the visual streak along the horizontal meridian is more prominent in the human than in the macaque retina (Stone and Johnston, 1981). Recent measurements in human visual cortex suggest that the volume representing the horizontal meridian is $\sim 3.5$ times larger than that representing the vertical meridian cortex (Janik et al., 2003). Together, these findings converge in supporting the notion of an overrepresentation of the horizontal relative to the vertical meridian. However, the magnitude of the anisotropy that we observed should be interpreted with caution, because our fMRI methodology cannot exclude many possible confounding factors, such as anisotropies in cell density, blood flow, or the response distribution of the population of neurons located within each single voxel.

\section{Functional subdivisions of the LGN}

We attempted to identify the $\mathrm{M}$ and $\mathrm{P}$ regions of the LGN based on their differences in sensitivity to stimulus contrast. Single-cell physiology studies in the macaque have shown that $\mathrm{M}$ cells have high contrast sensitivity (Derrington and Lennie, 1984; Sclar et al., 1990). We indeed observed a nonuniform distribution of contrast responses throughout the LGN and developed three criteria to consider LGN voxels to be candidates potentially containing significant fractions of $\mathrm{M}$ neurons: first, the voxels needed to be responsive to the $10 \%$ contrast stimuli; second, the voxels needed to exhibit only a small modulation in response amplitude evoked by the 10 and $100 \%$ contrast stimuli (CMI <0.25); and third, the voxels needed to be in the proper anatomical location.

We assumed that the voxels meeting the three criteria were the best candidate subregions within the LGN that may contain substantial fractions of M neurons. Those voxels that met our criteria for high contrast sensitivity and small differences in responses to high and low contrast stimuli tended to be clustered along the posterior, medial, and inferior surfaces of the LGN, the expected location of the M layers. Nineteen to $28 \%$ of the human LGN volume is occupied by the M layers (Andrews et al., 1997), which is similar to the $16.7 \%$ of LGN voxels identified as potential M voxel candidates using our functional criteria. It should be noted that our estimate depended on the choice of the activation and contrast modulation thresholds. Future studies using additional functional criteria will be necessary to further characterize the functional subdivisions within the human LGN.

The segregation of $\mathrm{M}$ and $\mathrm{P}$ divisions of the human LGN using functional criteria may be an exciting development toward probing visual functions of the two processing streams in relation to human behavior and cognition, because the LGN is the only location in the visual system in which the $\mathrm{M}$ and $\mathrm{P}$ channels are spatially segregated. Furthermore, the ability to directly assess the function of the M layers would be clinically useful, because abnormal development of the $\mathrm{M}$ layers is thought to occur in people with albinism (Guillery et al., 1975) and dyslexia (Livingstone et al., 1991; Stein, 2001).

\section{References}

Anderson RS, Wilkinson MO, Thibos LN (1992) Psychophysical localization of the human visual streak. Optom Vis Sci 69:171-174.

Andrews TJ, Halpern SD, Purves D (1997) Correlated size variations in human visual cortex, lateral geniculate nucleus, and optic tract. J Neurosci 17:2859-2868.

Azzopardi P, Cowey A (1996) The overrepresentation of the fovea and adjacent retina in the striate cortex and dorsal lateral geniculate nucleus of the macaque monkey. Neuroscience 72:627-639.

Bandettini PA, Jesmanowicz A, Wong EC, Hyde JS (1993) Processing strategies for time-course data sets in functional MRI of the human brain. Magn Reson Med 30:161-173.

Brainard DH (1997) The psychophysics toolbox. Spat Vis 10:433-436.

Brindley GS, Lewin WS (1968) The visual sensations produced by electrical stimulation of the medial occipital cortex. J Physiol (Lond) 194:54-55.

Brouwer B, Zeemann WBC (1926) The projection of the retina in the primary optic neuron in monkeys. Brain 49:1-35.

Chen W, Zhu X, Thulborn KR, Ugurbil K (1999) Retinotopic mapping of lateral geniculate nucleus in humans using functional magnetic resonance imaging. Proc Natl Acad Sci USA 96:2430-2434.

Clark WELG, Penman GG (1934) The projection of the retina in the lateral geniculate body. Proc R Soc Lond B Biol Sci 114:291-313.

Connolly M, Van Essen D (1984) The representation of the visual field in 
parvicellular and magnocellular layers of the lateral geniculate nucleus in the macaque monkey. J Comp Neurol 226:544-564.

Cowey A, Rolls ET (1974) Human cortical magnification factor and its relation to visual acuity. Exp Brain Res 21:447-454.

Creutzfeldt OD, Lee BB, Elepfandt A (1979) A quantitative study of chromatic organisation and receptive fields of cells in the lateral geniculate body of the rhesus monkey. Exp Brain Res 35:527-545.

Curcio CA, Allen KA (1990) Topography of ganglion cells in human retina. J Comp Neurol 300:5-25.

Curcio CA, Sloan KR, Kalina RE, Hendrickson AE (1990) Human photoreceptor topography. J Comp Neurol 292:497-523.

Daniel PM, Whitteridge D (1961) The representation of the visual field on the cerebral cortex in monkeys. J Physiol (Lond) 159:203-221.

Das A, Gilbert CD (1995) Long-range horizontal connections and their role in cortical reorganization revealed by optical recording of cat primary visual cortex. Nature 375:780-784.

Derrington AM, Lennie P (1984) Spatial and temporal contrast sensitivities of neurones in lateral geniculate nucleus of macaque. J Physiol (Lond) 357:219-240.

DeYoe EA, Bandettini P, Neitz J, Miller D, Winans P (1994) Functional magnetic resonance imaging (FMRI) of the human brain. J Neurosci Methods 54:171-187.

DeYoe EA, Carman GJ, Bandettini P, Glickman S, Wieser J, Cox R, Miller D, Neitz J (1996) Mapping striate and extrastriate visual areas in human cerebral cortex. Proc Natl Acad Sci USA 93:2382-2386.

Dougherty RF, Koch VM, Brewer AA, Fischer B, Modersitzki J, Wandell BA (2003) Visual field representations and locations of visual areas V1/2/3 in human visual cortex. J Vis 3:586-598.

Dreher B, Fukada Y, Rodieck RW (1976) Identification, classification and anatomical segregation of cells with X-like and Y-like properties in the lateral geniculate nucleus of old-world primates. J Physiol (Lond) 258:433-452.

Duncan RO, Boynton GM (2003) Cortical magnification within human primary visual cortex correlates with acuity thresholds. Neuron 38:659-671.

Engel SA, Rumelhart DE, Wandell BA, Lee AT, Glover GH, Chichilnisky EJ, Shadlen MN (1994) fMRI of human visual cortex. Nature 369:525.

Engel SA, Glover GH, Wandell BA (1997) Retinotopic organization in human visual cortex and the spatial precision of functional MRI. Cereb Cortex 7:181-192.

Erwin E, Baker FH, Busen WF, Malpeli JG (1999) Relationship between laminar topology and retinotopy in the rhesus lateral geniculate nucleus: results from a functional atlas. J Comp Neurol 407:92-102.

Grüsser OJ (1995) Migraine phosphenes and the retino-cortical magnification factor. Vision Res 35:1125-1134.

Guillery RW, Okoro AN, Witkop Jr CJ (1975) Abnormal visual pathways in the brain of a human albino. Brain Res 96:373-377.

Haacke EM, Hopkins A, Lai S, Buckley P, Friedman L, Meltzer H, Hedera P, Friedland R, Klein S, Thompson L, Detterman D, Tkach J, Lewin JS (1994) 2D and 3D high resolution gradient echo functional imaging of the brain: venous contributions to signal in motor cortex studies. NMR Biomed 7:54-62.

Hickey TL, Guillery RW (1979) Variability of laminar patterns in the human lateral geniculate nucleus. J Comp Neurol 183:221-246.

Hubel DH, Freeman DC (1977) Projection into the visual field of ocular dominance columns in macaque monkey. Brain Res 122:336-343.

Hubel DH, Wiesel TN (1974) Uniformity of monkey striate cortex: a parallel relationship between field size, scatter, and magnification factor. J Comp Neurol 158:295-305.

Janik JJ, Ropella KM, DeYoe EA (2003) Distortions of human retinotopy obtained with temporal phase mapped fMRI. Soc Neurosci Abstr 29:658.8.

Jenkinson M, Bannister P, Brady M, Smith S (2002) Improved optimisation for the robust and accurate linear registration and motion correction of brain images. NeuroImage 17:825-841.

Juba A, Szatmári A (1937) Ueber seltene hirnanatomische befunde in fällen von einseitiger peripherer blindheit. Klin Monatsbl Augenheilkd 99:173-188.

Kaas JH, Guillery WR, Allman JM (1972) Some principles of organization in the dorsal lateral geniculate nucleus. Brain Behav Evol 6:253-299.

Kaas JH, Huerta MF, Weber JT, Harting JK (1978) Patterns of retinal termi- nations and laminar organization of the lateral geniculate nucleus of primates. J Comp Neurol 182:517-553.

Kastner S, O'Connor DH, Fukui MM, Fehd HM, Herwig U, Pinsk MA (2004) Functional imaging of the human lateral geniculate nucleus and pulvinar. J Neurophysiol 91:438-448.

Kupfer C (1962) The projection of the macula in the lateral geniculate nucleus of man. Am J Ophthalmol 54:597-609.

Livingstone MS, Rosen GD, Drislane FW, Galaburda AM (1991) Physiological and anatomical evidence for a magnocellular defect in developmental dyslexia. Proc Natl Acad Sci USA 88:7943-7947.

Logothetis N, Merkle H, Augath M, Trinath T, Ugurbil K (2002) Ultra highresolution fMRI in monkeys with implanted RF coils. Neuron $35: 227-242$.

Mackenzie I (1934) Degeneration of the lateral geniculate bodies: a contribution to the pathology of the visual pathways. J Pathol 39:113-138.

Malpeli JG, Baker FH (1975) The representation of the visual field in the lateral geniculate nucleus of Macaca mulatta. J Comp Neurol 161:569-594.

Malpeli JG, Lee D, Baker FH (1996) Laminar and retinotopic organization of the macaque lateral geniculate nucleus: magnocellular and parvocellular magnification functions. J Comp Neurol 375:363-377.

Maunsell JH, Nealey TA, DePriest DD (1990) Magnocellular and parvocellular contributions to responses in the middle temporal visual area (MT) of the macaque monkey. J Neurosci 10:3323-3334.

Menon RS, Goodyear BG (1999) Submillimeter functional localization in human striate cortex using BOLD contrast at $4 \mathrm{~T}$ : implications for the vascular point-spread function. Magn Reson Med 41:230-235.

Merigan WH, Maunsell JH (1993) How parallel are the primate visual pathways? Annu Rev Neurosci 16:369-402.

Myerson J, Manis PB, Miezin FM, Allman JM (1977) Magnification in striate cortex and retinal ganglion cell layer of owl monkey: a quantitative comparison. Science 198:855-857.

O'Connor DH, Fukui MM, Pinsk MA, Kastner S (2002) Attention modulates responses in the human lateral geniculate nucleus. Nat Neurosci 5:1203-1209.

Pelli DG (1997) The VideoToolbox software for visual psychophysics: transforming numbers into movies. Spat Vis 10:437-442.

Perry VH, Cowey A (1985) The ganglion cell and cone distributions in the monkey's retina: implications for central magnification factors. Vision Res 25:1795-1810.

Polyak S (1953) Santiago Ramon y Cajal and his investigation of the nervous system. J Comp Neurol 98:3-8.

Rönne H (1910) Pathologisch-anatomische untersuchungen über alkoloische intoxikationsamblyopie. Arch Ophthalmol 77:1-95.

Rönne H (1913) Zur pathologischen anatomie der diabetischen intoxikationamblyopie. Arch Ophthalmol 85:489-537.

Rönne H (1914) Die anatomische projektion der macula im corpus geniculatum. Arch Psychiatr Nervenkr Z Gesamte Neurol Psychiatr 22:469-485.

Schein SJ, de Monasterio FM (1987) Mapping of retinal and geniculate neurons onto striate cortex of macaque. J Neurosci 7:996-1009.

Schwartz EL (1980) Computational anatomy and functional architecture of striate cortex: a spatial mapping approach to perceptual coding. Vision Res 20:645-669.

Sclar G, Maunsell JH, Lennie P (1990) Coding of image contrast in central visual pathways of the macaque monkey. Vision Res 30:1-10.

Sereno MI, Dale AM, Reppas JB, Kwong KK, Belliveau JW, Brady TJ, Rosen BR, Tootell RB (1995) Borders of multiple visual areas in humans revealed by functional magnetic resonance imaging. Science 268:889-893.

Shapley R, Kaplan E, Soodak R (1981) Spatial summation and contrast sensitivity of $\mathrm{X}$ and $\mathrm{Y}$ cells in the lateral geniculate nucleus of the macaque. Nature 292:543-545.

Slotnick SD, Klein SA, Carney T, Sutter EE (2001) Electrophysiological estimate of human cortical magnification. Clin Neurophysiol 112:1349-1356.

Stein J (2001) The magnocellular theory of developmental dyslexia. Dyslexia $7: 12-36$

Stone J, Johnston E (1981) The topography of primate retina: a study of the human, bushbaby, and new- and old-world monkeys. J Comp Neurol 196:205-223.

Talairach J, Tournoux P (1988) Stereotactic coplanar atlas of the human brain. New York: Thieme Medical. 
Talbot SA, Marshall WH (1941) Physiological studies on neuronal mechanisms of visual localization and discrimination. Am J Ophthalmol 24:1255-1263.

Thompson JK, Peterson MR, Freeman RD (2003) High resolution neurometabolic coupling in the lateral geniculate nucleus. Soc Neurosci Abstr 29:68.9.

Tootell RB, Silverman MS, Switkes E, De Valois RL (1982) Deoxyglucose analysis of retinotopic organization in primate striate cortex. Science 218:902-904.

Tootell RB, Switkes E, Silverman MS, Hamilton SL (1988) Functional anatomy of macaque striate cortex. II. Retinotopic organization. J Neurosci 8:1531-1568.

Tootell RB, Reppas JB, Kwong KK, Malach R, Born RT, Brady TJ, Rosen BR, Belliveau JW (1995) Functional analysis of human MT and related visual cortical areas using magnetic resonance imaging. J Neurosci 15:3215-3230.
Van Essen DC, Newsome WT, Maunsell JH (1984) The visual field representation in striate cortex of the macaque monkey: asymmetries, anisotropies, and individual variability. Vision Res 24:429-448.

Wässle H, Grunert U, Rohrenbeck J, Boycott BB (1989) Cortical magnification factor and the ganglion cell density of the primate retina. Nature 341:643-646.

Wässle H, Grunert U, Rohrenbeck J, Boycott BB (1990) Retinal ganglion cell density and cortical magnification factor in the primate. Vision Res 30:1897-1911.

Webb SV, Kaas JH (1976) The sizes and distribution of ganglion cells in the retina of the owl monkey, Aotus trivirgatus. Vision Res 16:1247-1254.

Wiesel TN, Hubel DH (1966) Spatial and chromatic interactions in the lateral geniculate body of the rhesus monkey. J Neurophysiol 29:1115-1156.

Woods RP, Grafton ST, Holmes CJ, Cherry SR, Mazziotta JC (1998) Automated image registration: I. General methods and intrasubject, intramodality validation. J Comput Assist Tomogr 22:139-152. 